\title{
Rivalités et arrangements coopératifs pour l'accès à l'eau souterraine dans la plaine de Berrechid au Maroc
}

\author{
Romaïssa Ouassissou ${ }^{1,2,3,{ }^{*}}$, Marcel Kuper ${ }^{1,2,3}$, Patrick Dugué ${ }^{2,3}$, Mohamed El Amrani ${ }^{4}$, Ali Hammani ${ }^{1}$ \\ et Fatah Ameur ${ }^{5}$ \\ ${ }^{1}$ Institut agronomique et vétérinaire Hassan II, Madinat Al Irfane, BP 6202, Rabat, Maroc \\ 2 CIRAD, UMR G-EAU, 73 rue Jean-François Breton, 34398 Montpellier, France \\ 3 G-EAU, Univ Montpellier, Montpellier, France \\ ${ }^{4}$ École nationale d'agriculture de Meknès, Km 10 route Haj Kaddour, BP S/40, Meknès 50001, Maroc \\ ${ }^{5}$ Centre de recherche en économie appliquée pour le développement, rue Djamel Eddine El-Afghani-El Hammadia, BP.197, Rostomia, \\ Bouzaréah, Alger, Algérie
}

\begin{abstract}
Résumé - Au Maroc, l'exploitation individuelle des eaux souterraines a permis le développement rapide d'une agriculture irriguée intensive. Dans la plaine de Berrechid, la ressource est limitée et surexploitée. L'agence de bassin tente depuis une dizaine d'années de mettre en place une contractualisation avec différents acteurs pour réguler les prélèvements en eau souterraine à usage agricole. L'objectif de l'étude est d'analyser en quoi la catégorisation des agriculteurs, se distinguant par leur accès à l'eau et au foncier, et les interactions entre ces catégories d'agriculteurs, sont utiles pour penser la gouvernance de la nappe. Une fois la dynamique de l'agriculture irriguée retracée, des enquêtes ont permis d'établir une typologie d'agriculteurs, puis les arrangements et rivalités entre les différents types d'agriculteurs ont été caractérisés. Les résultats montrent que l'utilisation accrue des eaux souterraines a déclenché des rivalités pour l'accès à l'eau, ainsi que l'exclusion de certains agriculteurs dans un contexte de baisse de la nappe. Pour dépasser ces problèmes d'accès, certains agriculteurs mettent en place des arrangements coopératifs autour des différents facteurs de production (eau, terre, capital, savoir-faire, main-d'œuvre). Cependant, ces arrangements accentuent, paradoxalement, la surexploitation de la nappe et les rivalités, et entraînent la poursuite et l'évolution des formes d'arrangements coopératifs. La compréhension fine de la nature des relations entre différents types d'agriculteurs peut permettre d'inclure une réflexion sur l'équité sociale dans le projet de concession aux associations de producteurs, qui pour le moment semble ignorer ces relations.
\end{abstract}

Mots clés : agriculture irriguée / surexploitation / exclusion / usagers / contrat de nappe

\begin{abstract}
Rivalries and cooperative arrangements for access to groundwater in the Berrechid plain in Morocco. In Morocco, private groundwater use enabled the rapid development of intensive irrigated agriculture. In the Berrechid plain, this resource is limited and overexploited. The river basin agency has attempted for the last ten years to set up a formal contract with various stakeholders to regulate agricultural use. The aim of the study is to analyze how the categorization of farmers, distinguished by their access to water and land, and the interactions between these categories are useful to think the governance of groundwater. The dynamics of irrigated agriculture were first traced and surveys enabled to establish a typology of farmers. Finally, the rivalries and the cooperative arrangements between different types of farmers were characterized. The results show that increased groundwater use has triggered rivalries for access to groundwater and the exclusion of some farmers in a context of falling water tables. To overcome these access problems, farmers set up cooperative arrangements around the factors of production required for irrigated agriculture (water, land, capital, know-how, labor). However, these arrangements accentuate, paradoxically, groundwater overexploitation and rivalries and lead to the continuation and change of the forms of cooperative arrangements. A detailed understanding of the nature of the relationships between the different types of farmers can address the issue of social equity in the concession contract with irrigation associations, which for the moment seems to ignore these relationships.
\end{abstract}

Keywords: irrigated agriculture / overexploitation / exclusion / users / aquifer contract

\footnotetext{
*Auteur de correspondance : romaissa.oua@gmail.com
} 


\section{Introduction}

Le développement de l'agriculture irriguée dans les zones semi-arides est très souvent lié à l'accès à l'eau souterraine permettant aux agriculteurs d'intensifier leurs productions (Moench, 2003). Cela explique l'engouement pour cette ressource et, par conséquent, la surexploitation des nappes dans de nombreux pays. Au Maroc, la surexploitation touche $50 \%$ des aquifères (Kuper et al., 2016). Dans la plaine de Berrechid, notre zone d'étude, l'agence du bassin hydraulique estime le bilan déficitaire annuel de la nappe à 30 millions de mètres cubes en 2016, cela étant lié aux prélèvements effectués à partir de quelques 3000 puits et forages, dont $96 \%$ du volume total pompé sont à usage agricole. L'utilisation croissante de l'eau souterraine est aussi le fruit d'une politique agricole ambitieuse, le Plan Maroc Vert, lancé en 2008 et procurant des subventions pour intensifier l'agriculture, en particulier le matériel d'exhaure et d'irrigation. Pour atténuer la pression exercée sur les nappes, l'agence de bassin tente depuis une dizaine d'années de mettre en place une contractualisation avec les différents acteurs pour réguler les prélèvements à usage agricole.

L'agriculture est souvent jugée comme «prédatrice» des eaux souterraines, étant responsable de $80 \%$ des prélèvements au niveau mondial (Famiglietti, 2014). Ce constat global masque cependant de grandes inégalités entre les différentes catégories d'exploitations agricoles en ce qui concerne leur contribution à la surexploitation des nappes et les bénéfices qu'elles en retirent (Ameur et al., 2017). En effet, l'accès à l'eau souterraine est souvent source de rivalités entre différentes catégories d'agriculteurs et est considéré comme un facteur de différenciation majeure des exploitations agricoles (Ameur et al., 2017). Si l'irrigation par les eaux souterraines est souvent considérée comme un catalyseur pour une transition socio-économique des agricultures vers un modèle agricole plus rémunérateur (Llamas et MartínezSantos, 2005), cette transition engendre aussi la marginalisation de certains agriculteurs qui n'ont pas les moyens financiers pour accéder aux eaux souterraines ou affronter la baisse des nappes (Mukherji, 2006). Ces rivalités sont souvent muettes et l'exclusion de l'accès aux eaux souterraines peut être interprétée comme une «fatalité produite par des mécanismes neutres et anonymes » dans un contexte de raréfaction de la ressource, alors qu'il s'agit souvent d'un processus de sélection et d'exclusion par la compétition économique (Del Vecchio et Mayaux, 2017).

Inversement, l'accès à l'eau souterraine peut être source de coopération. Shah (1993) et d'autres ont analysé les marchés informels de l'eau en Asie du Sud, qui ont permis à des agriculteurs dotés de faibles capitaux financiers d'accéder à l'eau souterraine. Au Maghreb, les formes de coopération entre agriculteurs pour l'accès à l'eau souterraine sont imbriquées dans un ensemble d'arrangements informels permettant de réunir les différents facteurs de production (eau, foncier, maind'œuvre, savoir-faire, capital) dans une même exploitation agricole. Ces arrangements améliorent l'accès à la nappe pour certains agriculteurs, mais n'assurent pas un accès généralisé et équitable aux différentes catégories d'agriculteurs et n'intègrent en aucun cas le souci de la préservation de la ressource (Boudjellal et al., 2011).

L'objectif de l'étude est d'analyser en quoi la catégorisation des agriculteurs - qui se distinguent par l'accès aux eaux souterraines et au foncier - et l'analyse des interactions entre ces catégories sont utiles pour penser la gouvernance de la nappe en vue d'une gestion plus durable de ce bien commun. La plaine de Berrechid au Maroc constitue un cas d'étude intéressant, car la ressource en eau y est limitée et exploitée par une grande diversité d'agriculteurs.

\section{Méthodologie}

La plaine de Berrechid, d'une superficie de $1500 \mathrm{~km}^{2}$, est située au sud de Casablanca (Fig. 1) et reçoit une pluviométrie annuelle inférieure à $400 \mathrm{~mm}$. Historiquement connue pour être l'un des greniers céréaliers du Maroc, elle subit depuis les années 1980 de plus en plus d'aléas pluviométriques. L'incertitude climatique affectant la production pluviale et la proximité de la ville de Casablanca ont incité certains agriculteurs à développer des cultures irriguées, en particulier le maraîchage et les cultures fourragères.

L'étude concerne deux villages ou douars de la commune Ouled Zidane, qui couvrent une superficie de 1046 ha. La partie la plus basse de la zone d'étude est appelée localement «Lhaoud», que l'on peut traduire par «bassin» (Fig. 1). Elle bénéficie d'apports d'eau par ruissellement et par les crues des oueds et se caractérise par la présence d'une nappe peu profonde $(40-60 \mathrm{~m})$.

Nous avons réalisé 15 entretiens collectifs avec les différents types d'agriculteurs pour reconstituer l'histoire de l'agriculture irriguée dans la commune. Ces entretiens ont été conduits dans des lieux d'échange des agriculteurs, comme le souk hebdomadaire, devant des minoteries et dans la commune rurale.

Ensuite, en combinant l'utilisation de Google Earth et d'un système d'information géographique, des enquêtes ont été réalisées auprès de tous les producteurs des deux douars. Nous avons cartographié les exploitations, précisé les assolements et localisé les ouvrages de pompage (profondeur, type d'ouvrage, année de creusement ou d'approfondissement ou de tarissement). Cela a permis de procéder à une classification typologique des 334 agriculteurs présents dans la zone, selon leur lieu de résidence, leur mode de faire-valoir, leurs activités agricoles dominantes (cultures pluviales ou irriguées et élevage), les superficies cultivées et le type d'accès à l'eau souterraine (puits ou forage ou puits-forage). Nous avons ensuite analysé les arrangements entre les différents types d'agriculteurs pour déterminer les catégories d'agriculteurs bénéficiant de l'eau souterraine et celles qui sont exclues. Nous avons interrogé l'ensemble des protagonistes des arrangements pour des raisons de triangulation de l'information.

\section{Résultats}

\subsection{Le développement de l'agriculture irriguée}

Avant les années 1990, la zone d'étude était connue pour ses productions céréalières (blé tendre et blé dur) et fourragères (orge), et son élevage ovin de race Serdi (Fig. 2). L'intensification des cultures pluviales était limitée par le faible revenu monétaire des exploitations, une bonne partie de la production étant destinée à l'alimentation familiale. La ressource hydrique souterraine était alors mobilisée à travers des puits traditionnels pour l'eau potable et l'abreuvement du cheptel. Elle était relativement abondante du fait de la recharge naturelle de la 


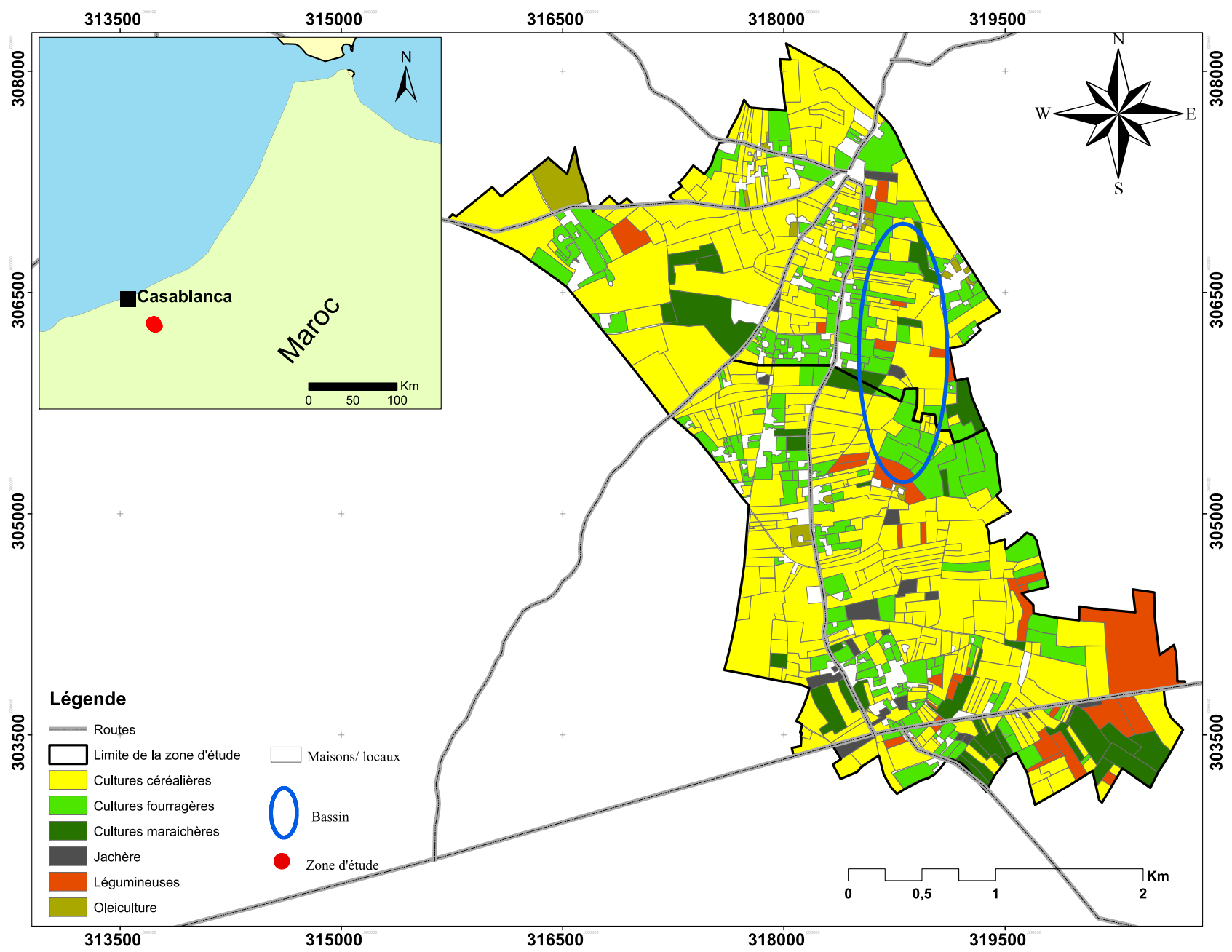

Fig. 1. Zone d'étude dans la plaine de Berrechid.

Fig. 1. The study area in the Berrechid plain.

nappe à partir des abouchements des oueds et la faible demande en eau. Ces puits permettaient aussi de cultiver la luzerne irriguée, à proximité des habitations, sur de très petites surfaces (environ 0,1 ha par exploitation).

Au début des années 1990, l'arrivée des agriculteurs locataires a entraîné le développement des cultures irriguées comme la pomme de terre et le maïs fourrager. Ces locataires étaient des producteurs non originaires de la zone, spécialisés dans la production maraîchère irriguée. En 2000, une partie des agriculteurs locaux ont développé les cultures maraîchères avec leurs propres moyens de production, via un processus d'apprentissage en travaillant chez les locataires comme ouvriers temporaires ou «en les regardant faire». Une partie des agriculteurs locaux, qui disposait ainsi désormais du savoir-faire, s'est associée avec de nouveaux producteurs locataires pourvoyeurs de capitaux financiers. Cela a constitué un nouveau mode de faire-valoir émergent à partir des années 2000, à travers des arrangements coopératifs (appelés localement contrats d'association) souvent informels au début, relatifs à différents facteurs de production. La loi sur l'eau 10-95 a été à l'origine de la création en 2000 de l'agence de bassin en charge de la plaine de Berrechid. Mais l'évolution des cadres juridique et institutionnel structurant la gestion de l'eau n'a pas encore permis le contrôle des usages qui alimentent désormais de nouvelles formes d'agricultures de plus en plus gourmandes en eau (Fig. 2).

Le développement de l'agriculture irriguée a engendré une évolution rapide du nombre et du type d'ouvrages de pompage (Fig. 3). En 1990, la zone d'étude comptait 93 puits d'une profondeur variant de 40 à $60 \mathrm{~m}$, alors que fin 2017, il y avait 219 points d'eau privés et 7 ouvrages publics (alimentation en eau de la commune, du lycée et de l'école). Ces 226 ouvrages sont répartis en 18 forages, 180 puits et 28 puits-forages (Fig. 3). Le puits-forage correspond au creusement d'un forage au fond d'un puits asséché ou à très faible débit. Sur un total de 219 ouvrages privés, seulement 41 puits et 14 forages sont utilisés pour l'irrigation. Parmi ceux-là, nous avons identifié les ouvrages les plus utilisés pour l'irrigation : 21 puits et 5 forages, qui sont utilisés de façon permanente et fournissent une grande part de l'eau d'irrigation pour des débits variant de 25 à $54 \mathrm{~m}^{3} / \mathrm{h}$ par ouvrage. Les autres ouvrages sont en majorité des puits dédiés à l'alimentation en eau potable et à l'abreuvement du bétail.

Cette évolution spectaculaire a bénéficié dans les années 1990 d'un programme de subvention pour le creusement des puits. Avant 2000, ce programme n'exigeait pas d'autorisation de 


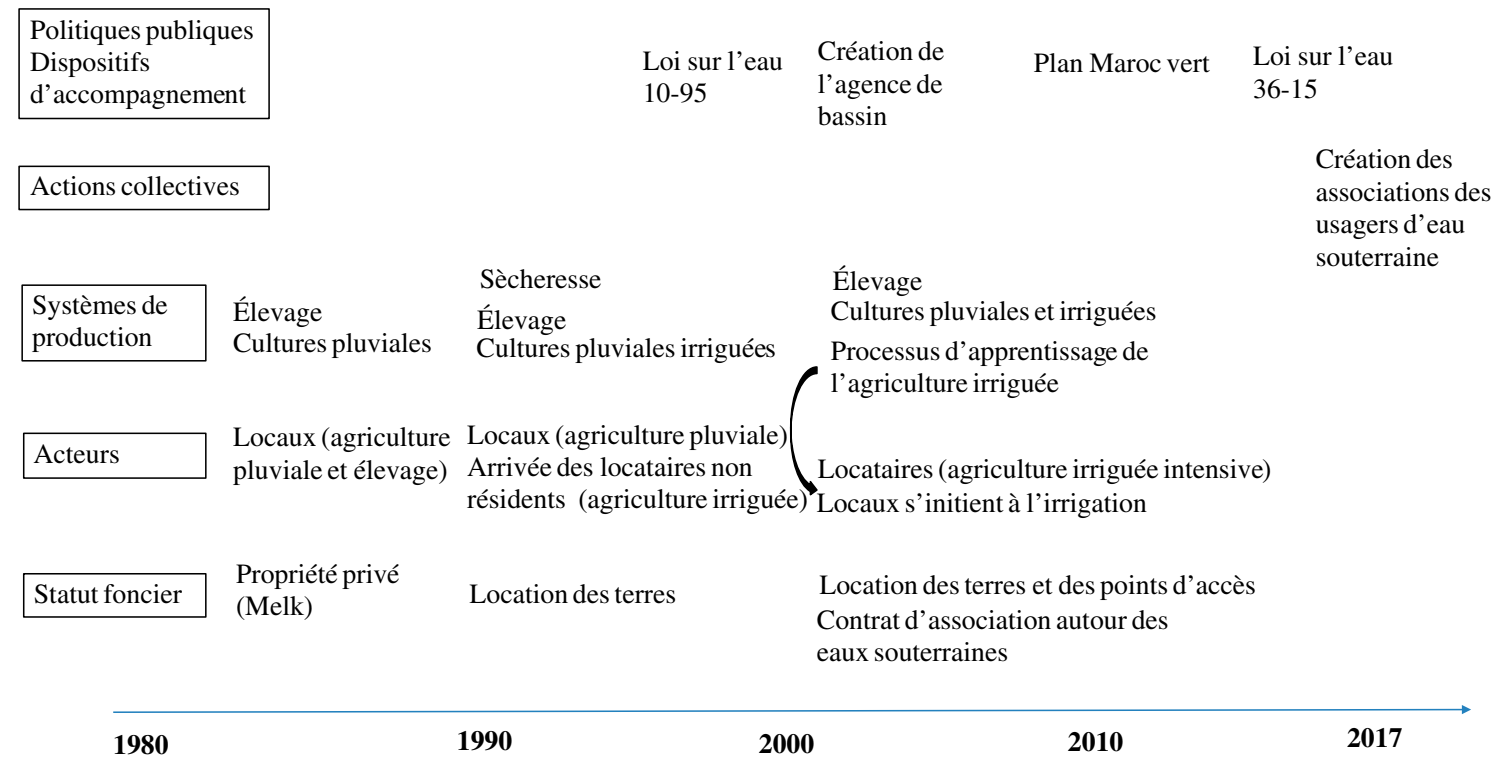

Fig. 2. Frise historique de l'agriculture irriguée.

Fig. 2. Historical timeline of irrigated agriculture.
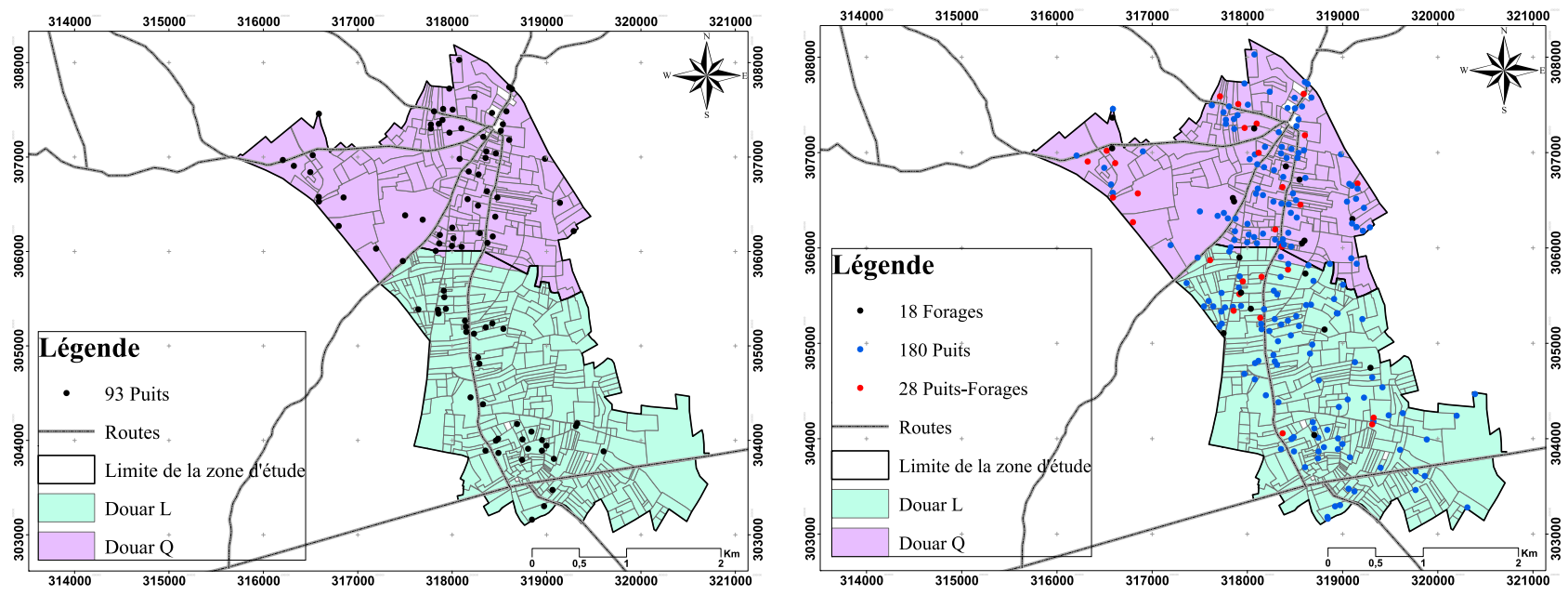

Fig. 3. Évolution des points d'accès à l'eau entre 1990 et fin 2017.

Fig. 3. Evolution of the access points to water between 1990 and 2017.

creusement ni de pompage pour obtenir la subvention, car les agences de bassin n'existaient pas encore et la loi sur l'eau 10-95 se mettait progressivement en place. La multiplication des puits et forages dans la plaine correspondait surtout à l'arrivée des locataires, venus des communes voisines ou des villes proches pour la production de cultures maraîchères et fourragères. Ils étaient pour la plupart d'origine rurale et étaient attirés par la proximité et la qualité de l'eau souterraine, la qualité du sol et la proximité des marchés des grandes villes. Ce mode de faire-valoir indirect a entraîné l'extension des surfaces irriguées et l'introduction du goutte-à-goutte. Pour les locataires, l'accès à la terre était nécessairement couplé à un accès aux eaux souterraines régulier et en quantité suffisante. Cela a généré le creusement de nouveaux ouvrages, le passage au forage et le surcreusement des points d'accès existants (Fig. 3).
Nos enquêtes ont montré que l'exploitation individuelle de l'eau souterraine pour l'irrigation, non régulée, a engendré une baisse continue de la nappe, le tarissement de 52 puits et l'effondrement de quelques forages. À cela s'ajoute la dégradation de la qualité de l'eau d'irrigation par un début de salinisation.

\subsection{Diversité des agriculteurs exploitant l'eau souterraine}

Malgré la forte progression des surfaces irriguées dans la zone d'étude, seule une petite minorité d'agriculteurs (19\%) bénéficie de l'eau souterraine pour l'irrigation. Vingt-six pourcent des 334 agriculteurs interrogés disposent d'ouvrages à faible débit qui leur permettent seulement d'abreuver leur 
bétail et de s'approvisionner en eau potable et $7 \%$ des agriculteurs détiennent des points d'accès désormais taris. En outre, $55 \%$ des agriculteurs dépendent pour leurs besoins domestiques et d'élevage des points d'eau publics ou de ceux des voisins.

La SAU de la zone d'étude est de 972 ha (sur 1046 ha) pour la campagne agricole 2016-2017, dont 79\% sont occupés par des cultures céréalières et fourragères conduites en bour (agriculture pluviale). Seuls $21 \%$ de la SAU sont irrigués. Les agriculteurs de la zone d'étude peuvent être répartis en quatre types selon leur accès à l'eau souterraine et selon le mode de faire-valoir (Tab. 1).

\subsubsection{Type 1: Agriculteurs locaux dont les exploitations sont conduites en agriculture pluviale}

Le type 1 compte 269 agriculteurs ( $80 \%$ du total), qui combinent les cultures pluviales avec l'élevage bovin et ovin. Ils détiennent une superficie de 575 ha en melk (propriété privée), dont 54 ha sont pris en location par 18 agriculteurs des types 3 et 4. Seulement 110 agriculteurs du type 1 ont un accès aux eaux souterraines via 94 points d'accès dédiés à l'abreuvement du bétail et à l'alimentation en eau potable. À cela s'ajoutent 9 ouvrages destinés à l'irrigation, dont 4 sont donnés en location aux producteurs des types 3 et 4 . L'accès à l'eau souterraine est limité pour ces agriculteurs, qui cultivent des céréales en bour sur une superficie de 520 ha, en particulier l'orge parce qu'elle est plus rémunératrice que le blé tendre $(40 €$ contre 20 à $25 € /$ quintal) et essentielle à leur élevage. Les parcelles d'orge (1 à 2 ha par exploitation) sont pâturées en janvier puis protégées du bétail jusqu'à maturité pour profiter de la vente des grains. La seconde céréale en superficie est le blé. Un agriculteur (Ahmed) âgé de 55 ans déclare que: «Le premier aliment que les gens servent sur la table est le pain, la pratique de la céréaliculture et de l'élevage comptent parmi les principes et les valeurs chez les vrais agriculteurs. L'agriculteur prépare le pain avec son propre blé, il ne l'achète pas». Les agriculteurs locaux s'identifient toujours aux cultures traditionnelles du territoire.

\subsubsection{Type 2: Agriculteurs locaux pratiquant l'agriculture irriguée}

Les agriculteurs du type 2 combinent des cultures en bour et irriguées, ainsi que l'élevage des ruminants. Cinquante agriculteurs (15\% de l'effectif total) disposent d'une superficie de 306 ha en propriété privée et donnent en location 7 ha à deux agriculteurs du type 3 ou 4. La superficie exploitée est partagée entre 268 ha de bour et 80 ha de cultures irriguées (maïs, pomme de terre). Ces agriculteurs mobilisent de plus en plus l'irrigation pour intensifier leurs systèmes de production. Une parcelle peut porter deux cultures par an avec de la pomme de terre irriguée (semis en février et récolte en juin), suivie de maïs irrigué (semis fin juillet début août et récolte en octobre). Ces agriculteurs exploitent 35 points d'eau pour l'irrigation dont 11 forages, et ils mettent 4 puits à disposition des locataires à travers des arrangements.

\subsubsection{Type 3: Agriculteurs locaux prenant en location des terres et pratiquant l'agriculture irriguée}

Le type 3 comprend 10 agriculteurs ( $4 \%$ ), qui combinent cultures pluviales et irriguées. Ils maîtrisent bien la conduite technique du maraîchage irrigué, accumulant un savoir-faire depuis deux décennies. Ils résident sur place et portent le flambeau de l'innovation dans la zone, car ils entretiennent des relations avec toutes les autres catégories de producteurs. Soixante et onze pourcent (ou 84 ha) des terres qu'ils cultivent sont en propriété privée (Tab. 1). Afin d'accroître leurs revenus, ils prennent en location des terres fertiles avec un accès à l'eau (41 ha en 2016-2017). La durée des contrats de location dépasse rarement un an. Un agriculteur (Abdesamad) âgé de 39 ans dit avec fierté : " grâce à la pomme de terre, j'ai pu acheter une voiture de marque à 14 millions de centimes [environ 14000 euros] cash sans recourir au crédit». Trois agriculteurs (sur 10) s'associent à des personnes étrangères aux douars (type 4), qui apportent le financement des intrants et de la main-d'œuvre. Ces derniers maîtrisent bien les calculs économiques et la commercialisation des produits maraîchers, car ils sont généralement commerçants en contact avec des détaillants et consommateurs. Ils s'associent aux agriculteurs locaux, qui maîtrisent mieux le savoir-faire lié à la production.

\subsubsection{Type 4: Locataires non-résidents et mobiles}

Le type 4 concerne seulement 5 agriculteurs ( $1 \%$ ) qui ne résident pas sur place, mais généralement dans les douars ou les villes proches. Ils sont considérés comme les fondateurs de l'agriculture irriguée dans la zone (Fig. 2). Leurs pratiques agricoles et d'irrigation ont été reprises par les agriculteurs locaux. Les terres cultivées par ces producteurs ont été louées auprès des locaux.

Certains locataires de ce type sont aussi des commerçants grossistes et spéculateurs qui achètent la pomme de terre chez des agriculteurs et la stockent en unités frigorifiques afin d'en tirer un meilleur prix en fonction des fluctuations du marché. Pour accroître leurs stocks, ils ont commencé à cultiver la pomme de terre vers 2003. Ces agriculteurs commerçants, sans liens historiques avec les deux douars, ont accès à la terre et à l'eau d'irrigation uniquement à travers des contrats de location et des arrangements. Ils apportent le capital nécessaire à la production et s'occupent eux-mêmes de la commercialisation en ville. La production est assurée par les agriculteurs locaux expérimentés dans la culture de la pomme de terre à travers des arrangements entre producteurs. Ces producteurs locataires sont très mobiles en fonction des opportunités de location de la terre. Ils cultivent dans différentes régions du Maroc. D'autres locataires proviennent du milieu rural des communes voisines et ont accumulé de l'expérience en agriculture irriguée depuis 1990. Ils sont venus à Ouled Zidane car ils étaient confrontés à la dégradation de la qualité du sol et au rabattement de la nappe sur les terres qu'ils occupaient. Leur profil se rapproche du type 3, car ils sont présents sur le terrain pour surveiller la production agricole.

\subsection{Arrangements et rivalités pour l'accès aux facteurs de production}

Nous avons identifié trois formes de coopération entre agriculteurs. La première est une transaction par laquelle les agriculteurs de type 1 et 2 , disposant de plus de terres qu'ils ne peuvent en cultiver ou d'un point d'eau non exploité pour l'irrigation, mettent en location ces facteurs de production afin d'en tirer un revenu annuel régulier (Fig. 4). Le prix de location 
R. Ouassissou et al. : Cah. Agric. 2019, 28, 4

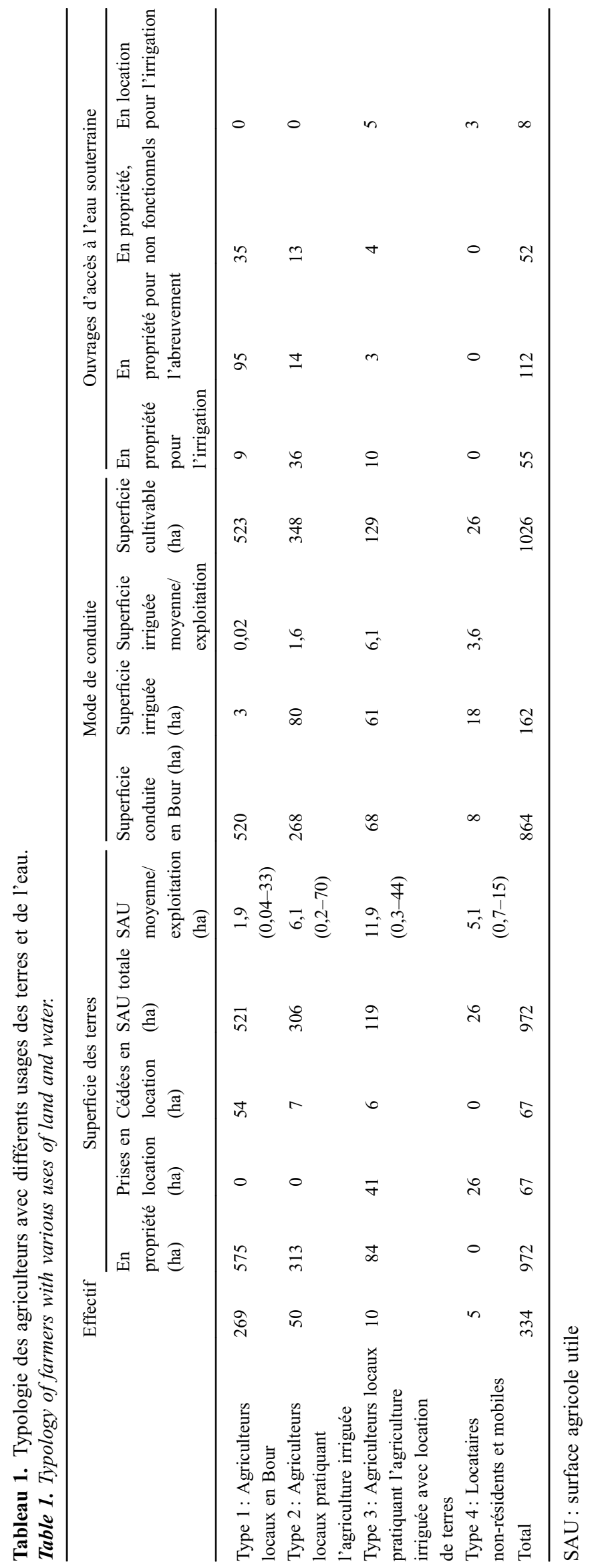

Page 6 de 10 
d'un hectare pour la durée d'un cycle cultural en culture pluviale varie entre 250 et $500 €$, tandis que celui d'un hectare équipé d'un puits fonctionnel est compris entre 600 et $800 €$. La location d'un puits ou forage avec l'accès à l'eau varie, lui, entre 1500 et $2500 €$ pour une campagne agricole, selon le rendement de l'ouvrage qui se traduit par des durées et des débits de pompage importants.

Les deux autres formes de coopération relèvent d'arrangements coopératifs à l'échelle de l'exploitation agricole. En effet, un agriculteur qui, à un moment donné, ne dispose pas de l'ensemble des facteurs de production nécessaires cherchera à obtenir les facteurs qui lui manquent auprès d'autres acteurs à travers les arrangements.

Le premier type d'arrangement se présente quand des agriculteurs locaux (type 1, 2 et 3 ), ayant acquis une bonne technicité en culture irriguée, s'associent à des locataires (types 3 et 4). Ces derniers accèdent au foncier et à l'eau à travers la location et apportent le capital nécessaire à la production agricole. Les agriculteurs locaux apportent leur savoir-faire et leur force de travail pour la production irriguée. De plus, ils mobilisent leurs réseaux socio-professionnels pour identifier des terres et des points d'accès à l'eau susceptibles d'être loués et surtout ayant un bon rendement. Ce sont eux qui mènent les négociations pour le compte de leurs associés. Ce type d'arrangement permet aux jeunes de la commune de développer une activité productive localement au lieu de migrer vers d'autres régions.

Un deuxième type d'arrangement concerne les locataires résidents (type 3), qui sont en mesure de prendre en location des terres (auprès des types 1 et 2 ) et de produire, tout en obtenant du capital des locataires mobiles (type 4). Ces acteurs financent les arrangements, mais pratiquent aussi l'agriculture intensive dans d'autres régions. Ils sont très mobiles et généralement absents sur le terrain. Par ailleurs, les protagonistes de ces arrangements sont très discrets, comme le remarque un agriculteur de la zone: «ces financeurs se cachent derrière les locataires résidents et ces derniers ne déclarent pas que le financement est issu de ces acteurs ».

La nature des arrangements est très évolutive. Premièrement, l'accès à l'eau devient difficile avec la baisse de la nappe, qui se traduit par un plus faible nombre d'ouvrages de pompage performants. Deuxièment, la contrainte agronomique pousse les locataires à changer de parcelles annuellement, car ils ne peuvent pas produire de la pomme de terre ou de la carotte deux années successives sur une même parcelle. Troisièment, la pression foncière dans la zone a fortement augmenté avec le développement de l'agriculture irriguée. Cela amène les locataires à trouver des parcelles de plus en plus loin des points d'accès à l'eau. Dans ce cas, les locataires installent des conduites en PVC pour amener l'eau sur des distances allant jusqu'à $5 \mathrm{~km}$.

Les arrangements coopératifs permettent de créer de la richesse, tout en ayant des effets directs et indirects sur les ressources. Ces effets varient selon les catégories d'agriculteurs. Le contrat d'association (oral ou formalisé) est à évaluer avec attention, car les agriculteurs locaux qui supervisent la production ne gagnent généralement que la moitié du revenu issu de la parcelle. Les locataires non résidents qui apportent le capital percevront l'autre moitié du gain. Cette mise en synergie des capitaux des différents acteurs entraîne aussi une dégradation de l'état des ressources naturelles: le déficit hydrique au niveau de la nappe s'accentue du fait de l'approfondissement des points d'eau et de l'accroissement des surfaces irriguées; l'entretien de la fertilité du sol est mal assuré par les locataires, car ils optent plutôt pour une stratégie minière de court-terme, en changeant de parcelle tous les ans. Les arrangements sont habituellement informels et reposent sur la confiance et l'oralité. Mais certains contrats d'association ont été récemment « officialisés » par un écrit signé par les deux parties et visé par l'administration communale, même si sur le plan légal ces documents n'ont pas de valeur juridique. Cette évolution correspond à une distanciation sociale accrue entre parties prenantes. Comme le dit un agriculteur: «L'absence du contrat peut engendrer des problèmes... Le contrat est une preuve en cas de décès d'une des parties signataires. Dans ce cas, le contrat reste comme témoin pour éviter toute forme d'expropriation du bien ».

Une partie des producteurs locaux de la zone profite du marché de la terre et de l'eau: «L'eau souterraine devient de plus en plus rare, mais nous ne pouvons pas manifester contre ces locataires parce qu'il y a des "Ouled Lbled" [litt. : les fils de la zone] maîtrisant bien les cultures irriguées qui s'associent avec eux pour obtenir des intrants et des équipements, et d'autres agriculteurs locaux qui tirent profit de la location de leurs terres et de leurs puits pour assurer leurs besoins quotidiens ». Ainsi, les agriculteurs de type 1 et 2 donnent en location 8 puits agricoles qui peuvent atteindre un débit de $50 \mathrm{~m}^{3} / \mathrm{h}$ chacun et une surface de 61 ha auprès des producteurs locataires (types 3 et 4 ).

Mais le développement de l'agriculture irriguée entraîne aussi des conflits relatifs à l'usage de l'eau souterraine, des espaces pastoraux traditionnels et de la vaine pâture (Fig. 4). Les agriculteurs locaux pratiquant l'irrigation (type 2) se plaignent des locataires (types 3,4 ), qui prélèvent de grandes quantités d'eau du fait de leurs grandes surfaces irriguées. Ils les accusent de «sucer notre eau» et de tirer profit de ce bien commun. En particulier, les locataires «nomades», venant de l'extérieur de la zone, sont souvent montrés du doigt dans les discussions autour des prélèvements d'eau agricole. De plus, les agriculteurs en bour pratiquant l'élevage (type 1) voient se réduire les espaces de vaine pâture après les récoltes de céréales suite à l'extension des surfaces irriguées. À cela s'ajoute le fait que la baisse de la nappe a entraîné le tarissement de 52 puits peu profonds destinés à l'alimentation en eau potable et à l'abreuvement de bétail. Ces tensions autour de l'accès aux ressources productives et à l'eau potable renforcent le sentiment d'exclusion et de marginalisation des agriculteurs qui ne peuvent pas suivre la course d'exploitation de l'eau souterraine. Ces agriculteurs ont de trop petites surfaces $(<1$ ha) pour bénéficier du marché de la location des terres, car les locataires s'intéressent à des parcelles supérieures à 2 ha (Fig. 4).

\section{Discussion et conclusion}

\subsection{Des formes de coopération générant exclusion et épuisement des ressources}

Nous avons caractérisé un ensemble d'arrangements coopératifs permettant aux agriculteurs soit d'accéder à des facteurs de production (terre, eau, savoir-faire, capital, intrants et main-d'œuvre) pour mettre en place des systèmes de 

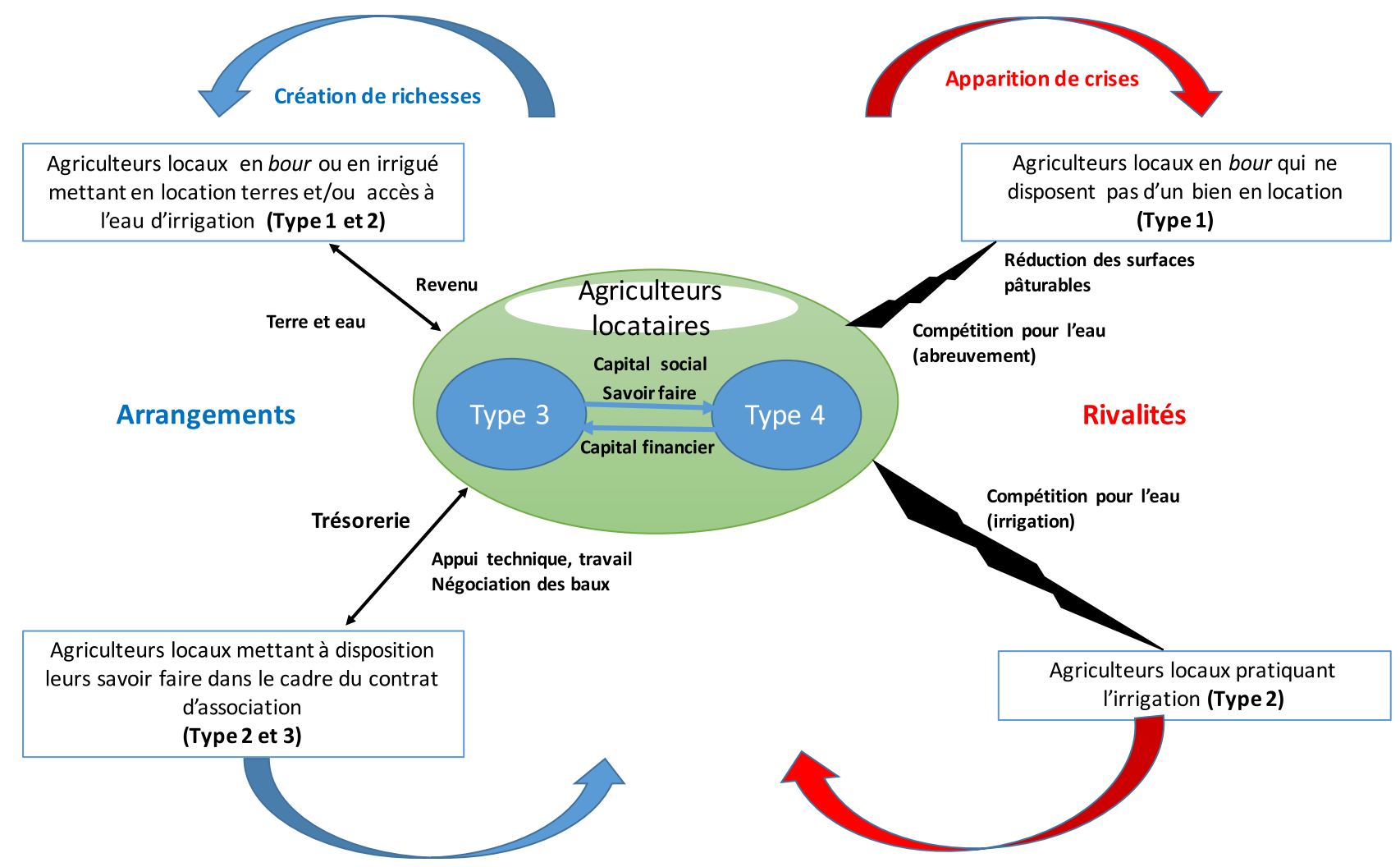

Fig. 4. Schéma illustratif des arrangements et des rivalités entre acteurs.

Fig. 4. Illustrative diagram of arrangements and rivalries between stakeholders.

production diversifiés, soit d'obtenir un revenu complémentaire. La confrontation à des situations de production contraintes donne naissance à ces arrangements informels. Le développement d'une agriculture intensive à partir des eaux souterraines a entraîné une augmentation de ce type d'arrangements au Maghreb et, plus important encore, les relations entre parties prenantes ont changé en faveur de celui qui détient le capital (Ameur et al., 2017). Certes, ces arrangements permettent aux agriculteurs locaux de maintenir leurs activités traditionnelles en agriculture pluviale, et aux familles rurales de garder une partie de leurs terres tout en recevant un revenu via la location. Mais au fil des années, les locataires (non) résidents ont construit un quasi-monopole d'accès à l'eau souterraine, s'accaparant la presque-totalité des richesses générées.

À Berrechid, les agriculteurs qui ne disposent ni du capital, ni d'un bien à donner en location, ni du savoir-faire qui les rend incontournables pour des locataires, sont condamnés à l'exclusion. Leur exclusion relève donc d'une incapacité de participer aux arrangements coopératifs. Cette exclusion concerne surtout les agriculteurs du type $1: 251$ sur 269 agriculteurs de ce type non seulement n'ont pas accès à l'eau souterraine, mais se retrouvent aussi exclus des arrangements et des transactions. L'exclusion concerne également à court ou moyen terme les agriculteurs du type 2 en raison de la baisse de la nappe. Cela révèle les limites des arrangements dans leur dimension entrepreneuriale autour des facteurs de production. Dans certains cas spécifiques, il peut y avoir des formes d'arrangements solidaires entre irrigants, afin de sauver leurs cultures par des arrangements ponctuels (Boudjellal et al., 2011).
La logique des arrangements, conçus pour dépasser à court-terme des contraintes d'accès aux ressources productives, accorde peu de considération à la pérennité de l'eau souterraine ou la fertilité des sols. Les locataires cherchent à garantir de hauts rendements par une sur-irrigation et pratiquent une fertilisation uniquement minérale peu respectueuse du sol, ce qui induit des conflits avec les propriétaires terriens qui veulent préserver la fertilité de leur sol. Cependant, les pratiques agricoles intensives, au départ propres aux locataires, sont de plus en plus reprises par l'ensemble des types d'agriculteurs.

Cette étude montre la fragilité de la majorité des exploitations familiales de la zone d'étude, et leur participation limitée au développement économique du territoire. Ces agriculteurs sont devenus dépendants des revenus liés à la location de la terre et des points d'eau. En outre, les locataires peuvent se retirer de la zone dès qu'elle ne leur apparaît plus suffisamment rémunératrice, par exemple en cas de baisse de fertilité des sols ou du niveau de la nappe. Au Maghreb, ces mécanismes d'exclusion des petits agriculteurs au profit des locataires concourent à la même tendance de prélèvement accru des eaux souterraines par une minorité de producteurs et de baisse généralisée des nappes (Ameur et al., 2017). De plus, ils génèrent de nouvelles relations de pouvoir et de nouveaux rapports de force entre catégories d'agriculteurs, en faveur du facteur capital, qui ne vont pas faciliter la mise en place et l'application de modalités de régulation des prélèvements d'eau souterraine, et donc de gestion raisonnée des aquifères.

En résumé, le phénomène d'exclusion prend trois formes. La première forme concerne les agriculteurs locaux pratiquant 
les cultures pluviales et l'élevage (type 1). La plupart de ces agriculteurs ne disposent ni du capital pour sur-creuser leurs ouvrages, ni de terres à donner en location pour avoir des revenus supplémentaires. La deuxième forme touche les agriculteurs locaux pratiquant des cultures irriguées et pluviales diversifiées (type 2). Un agriculteur déclare: «avec la présence des locataires, qui ne laissent pas leurs ouvrages de pompage en pause, à cause des grandes superficies de pomme de terre et de maïs fourrager qu'ils doivent irriguer, on souffre d'une baisse du niveau de l'eau dans nos puits ». Cette exclusion se traduit par le tarissement de 52 puits et l'approfondissement de 28 puits. La troisième forme d'exclusion concerne les locataires «nomades», qui sont exclus socialement par une partie des autochtones. Ces derniers les désignent comme les seuls prédateurs des eaux souterraines. Cette troisième exclusion explique pourquoi certains arrangements les concernant sont dissimulés.

\subsection{Deux logiques opposées: contrat de nappe et arrangements coopératifs}

L'exploitation minière des eaux souterraines à des fins d'irrigation dans la plaine de Berrechid a poussé l'agence à travailler depuis plus de 10 ans sur l'élaboration d'un contrat de nappe. Ce contrat devra associer divers acteurs publics et des associations de producteurs. L'objectif affiché du contrat est de limiter les prélèvements à 70 millions de mètres cubes/an à l'horizon 2035. Si aucune action n'est entreprise, la nappe de Berrechid connaîtra une augmentation des prélèvements de $5 \%$ par an jusqu'en 2035 selon l'agence de bassin. Cet objectif de réduction des prélèvements devrait accentuer les rivalités entre usagers de l'eau. L'agence envisage d'établir un contrat de concession en confiant la gestion des prélèvements d'eau agricole à des associations d'usagers des eaux souterraines. Ces associations devront inclure les grands consommateurs d'eau souterraine, en particulier les locataires (types 3 et 4). L'efficacité du contrat de nappe pour réduire les prélèvements implique une action collective engageant tous les irrigants, quelle que soit leur stratégie économique. Mais cet objectif ambitieux va non seulement à l'encontre des stratégies individuelles des agriculteurs, mais aussi de la logique des arrangements coopératifs de dépasser toute contrainte à court terme relative aux facteurs de production, conduisant à valoriser l'eau d'irrigation jusqu'à son épuisement. La logique de ces arrangements est souvent justifiée en faisant référence aux politiques agricoles actuelles misant sur une intensification de l'agriculture. Dans un contexte de baisse de la nappe, elle se heurte à la logique du projet de concession qui a mis la préservation de la nappe au centre des débats, entraînant nécessairement la réduction des prélèvements agricoles, mais remettant également en cause la logique des arrangements coopératifs.

\subsection{Implications pour la gouvernance de l'eau souterraine}

Au-delà du simple constat d'un fossé entre les aspirations à une gestion plus durable de la nappe et les pratiques agricoles actuelles, nous voulons tirer de cette étude les enseignements d'une situation de rivalités et de formes de coopération.
Le premier enseignement concerne les rivalités existantes entre différentes catégories d'agriculteurs. L'agence souhaite que tous les irrigants déclarent leurs assolements en début de campagne, ainsi que les prélèvements d'eau effectués. Réunir les différentes catégories d'agriculteurs dans une seule association d'usagers de l'eau souterraine est difficile. Les agriculteurs du type 3 sont déjà en position de force dans les arrangements avec les autres agriculteurs (types 1,2 ) et se positionnent comme les futurs responsables de ces associations. Un autre problème réside dans l'intégration de la catégorie des locataires non résidents (type 4), qui manifestent peu d'intérêt pour la bonne gestion des ressources naturelles et la durabilité de l'agriculture. Enfin, est-il juste d'exclure des discussions les agriculteurs non-irrigants, qui n'arrivent pas à accéder à la nappe pour l'irrigation et qui éprouvent même des difficultés croissantes pour s'approvisionner en eau potable et abreuver leur cheptel (type 1)?

Le deuxième enseignement des formes actuelles de coopération consiste à penser que si la gouvernance des eaux souterraines est entièrement déléguée aux seules associations présidées par les locataires résidents, il est peu probable que les questions d'exclusion et de durabilité de la ressource soient traitées. Le rôle de la puissance publique, à travers l'agence de bassin, va être déterminant pour la prise en compte d'une forme d'intérêt général. Mais comment l'agence peut-elle assurer à la fois le contrôle et le respect du contrat de nappe tout en déléguant la gestion de l'eau aux associations?

Le troisième enseignement est que les formes actuelles de coopération entre locataires et agriculteurs locaux montrent les prémices d'une nouvelle étape de partenariat et le développement de capacités d'innovation organisationnelle. Mais comment valoriser ces capacités pour développer l'action collective pour une gestion raisonnable de la nappe sans exclure les agriculteurs souhaitant maintenir une diversité d'activités productives (élevage, cultures pluviales)? Avant cela, les acteurs publics devraient commencer par reconnaître ces formes de coopération qui restent pour eux invisibles, car informelles, et peu prises en compte dans l'élaboration du contrat de nappe.

\section{Références}

Ameur F, Kuper M, Lejars C, Dugue P. 2017. Prosper, survive or exit: Contrasted fortunes of farmers in the groundwater economy in the Saiss plain (Morocco). Agricultural Water Management 191: 207 217. DOI: 10.1016/j.agwat.2017.06.014.

Boudjellal AA, Bekkar Y, Kuper M, Errahj M, Hammani A, Hartani T. 2011. Analyse des arrangements informels pour l'accès à l'eau souterraine sur les périmètres irrigués de la Mitidja (Algérie) et du Tadla (Maroc). Cahiers Agricultures 20(1-2): 85-91. DOI: 10.1684/agr.2010.0458.

Del Vecchio K, Mayaux PL. 2017. Gouverner les eaux souterraines au Maroc. L'État en aménageur libéral. Gouvernement et Action Publique 6(1): 107-130. DOI: 10.3917/gap.171.0107.

Famiglietti JS. 2014. The global groundwater crisis. Nature Climate Change 4(11): 945-948 DOI: 10.1038/nclimate2425.

Kuper M, Faysse N, Hammani A, Hartani T, Marlet S, Hamamouche F, et al. 2016. Liberation or Anarchy? The Janus nature of groundwater use on North Africa's new irrigation frontiers. In: Jakeman AJ, et al., ed. Integrated Groundwater Management: Concepts, Approaches and Challenges. Dordrecht, The Nether- 
lands: Springer Open, pp. 583-615. DOI: 10.1007/978-3319-23576-9.

Llamas M, Martínez-Santos P. 2005. Intensive groundwater use: silent revolution and potential source of social conflicts. Journal of Water Resources Planning and Management 131 (5): 337-341. DOI: 10.1061/(ASCE)0733-9496(2005)131: 5(337).

Moench M. 2003. Groundwater and poverty: exploring the connections. In: Llamas $\mathrm{R}$, et al., eds. Intensive Use of
Groundwater: Challenges and Opportunities. Lisse, The Netherlands: Swets et Zeitlinger B.V, pp. 441-456.

Mukherji A. 2006. Political ecology of groundwater: The contrasting case of water-abundant West Bengal and water-scarce Gujarat, India. Hydrogeology Journal 14(3): 392-406. DOI: 10.1007/ s10040-005-0007-y.

Shah T. 1993. Groundwater markets and irrigation development: Political economy and practical policy. Bombay: Oxford University Press, $241 \mathrm{p}$.

Citation de l'article : Ouassissou R, Kuper M, Dugué P, El Amrani M, Hammani A, Ameur F. 2019. Rivalités et arrangements coopératifs pour l'accès à l'eau souterraine dans la plaine de Berrechid au Maroc. Cah. Agric. 28: 4. 